\title{
Performance Optimization of Tethered Balloon Technology for Public Safety and Emergency Communications
}

\author{
S. H. Alsamhi ${ }^{1,2,3}$, F. A. Almalki ${ }^{4}$, Ou Ma ${ }^{5}$, M. S. Ansari ${ }^{1}$, M. C. Angelides ${ }^{4}$ \\ ${ }^{1}$ School of Aerospace Engineering, Tsinghua University, Beijing, China \\ ${ }^{2}$ Department of Electronics Engineering, Aligarh Muslim University, India \\ ${ }^{3}$ IBB University Yemen \\ ${ }^{4}$ Department of Electronic and Computer Engineering, Brunel University London, Uxbridge, UK \\ ${ }^{5}$ College of Engineering and Applied Science, University of Cincinnati, Ohio, USA \\ s.alsamhi.rs.ece@iitbhu.ac.in; faris.almalki@brunel.ac.uk; ou.ma@uc.edu; samar.ansari@zhcet.ac.in; \\ marios.angelides@brunel.ac.uk;
}

\begin{abstract}
This paper investigates scheme of Tethered Balloon network architecture based public safety network and emergency communications. Recently, Tethered Balloon technology represents the most powerful and promising technology to improve the public safety and saving people lives. The capability of network architecture enables Tethered Balloon to accomplish unique and specific missions for temporary events such as natural hazardous and terrorist acts. Natural hazardous and terrorist acts have significant potential to disrupt the entire terrestrial wireless communication infrastructure. In order to mitigate the effects of catastrophic, we propose Tethered Balloon as the key technology for delivering broadband services where communication infrastructures are partially or totally destroyed. This work is done by using OPNET Modeler 14.5 for network implementation. The result is shown the significant performance of using proposed technology to provide high priority for disaster area and deliver broadband communication services. It proves that rescue teams have significantly performed their duty effectively and efficiently.
\end{abstract}

Keywords: Public safety network, Tethered Balloon, Emergency services, OPNET, WiMAX, Hazardous, Disaster recovery.

\section{Introduction}

The weak point of traditional wired and wireless communication network technologies (e.g. 2G, 3G, 4G) is that they are easy disablement by natural disasters (e.g. earthquake, flood, tornado), or terrorist attacks. Many natural disasters created such as tsunami in Indonesia, earthquake in Pakistan and Venchuan in China, and Hurricane Katrin in the US, the bomb blasts in the cinema halls of Delhi and Chapala flood in Socotra Island in Yemen and etc. These mentioned events have diverted and take our focus towards the safety of people lives. During disasters, telecommunications infrastructure and the emergency communication operations might be unavailable due to the destroyed area. To establish an effective and efficient communication services in the large disaster area, a high bandwidth wireless solution is the most highly recommended [1].

Broadband communication services is able to make 911 and emergency systems during and aftermath the disaster more capable for providing better protection of 
people lives. Public safety answering points could receive pictures, text, videos from public and rebroadcasting them to first responders. Broadband communication technologies for public safety communications systems and emergency services have several benefits such as allows first responders in anywhere to receive and send data, voice and video to save people lives, prevent the terrorist acts, ensure all of people can access emergency services quickly, and etc. Generally, the efficient and reliable communication plays pivotal role in public safety network and particularly for disaster recovery and emergency response operations. During and after disaster, the main issue is the absence the terrestrial communications infrastructure equipment which is used by different public safety networks. Therefore, the emergency services and plagued rescue and relief teams are unable to perform their tasks properly.

Nowadays, traditional wireless communication solution could not be capable of delivering the services to cover the complex emergency situations public safety networks, which are being a wide area. The key factors for public safety and emergency communication services are immediate availability, rapid deployment, and reliability. However, an efficient communication unit was able to assess the communication needs quickly and restore basic communication infrastructure within a short period. During large disaster area, it is required for week or month for cellular communication providers to be recovered. Hence, terrestrial wireless communication networks are limited in term of capacity, coverage while terrestrial wireless communication may have been destroyed [2]. Therefore, space communication technologies play a fundamental role for disaster mitigation and recovery. Furthermore, space technologies are the essential tool for collecting information needs to protect human and mitigate economic losses due to disasters. In the immediate aftermath of an emergency disasters, Satellite represented as one of the reliable solution [3]. However, the specific result requirements were not present in this case. Besides, manufacturing, launching cost and delay are the weakness of communication via satellite.

The newest space technology is aerial platforms, which includes the advantages of both terrestrial and satellite systems. There are three categories of aerial platform: High Altitude Platform (HAP), Medium Altitude Platform (MAP) and Low Altitude Platform (LAP) [my hap and applications]. HAP offers an excellent alternative to provide emergency telecommunications after a catastrophic 
incident [4]. WiMAX connectivity from HAP was a novel approach. Moreover, some research studies have already been made in order to show the performance, operation and effectiveness of WiMAX connectivity through HAP payload [5-7].

Recently, Tethered Balloon concept (from one of the LAP family) operates at 200-440 m altitude above the ground surface [8,9]. It has significant advantages like low deployment cost, line of sight, rapid deployment, low propagation delay, fixed position and especially used for disasters recovery [10]. Tethered Balloon is the key technology for emergency situations and plays vital role for natural and human-induced disasters [10]. It becomes also the key technology for rapid deployable broadband wireless communication networks. Furthermore, It is capable of deliver wireless communication services for public safety after the catastrophic. Therefore, Tethered Balloon can be considered as an important part for reliable public safety network and save people lives. The capability of tethered balloon enables Tethered Balloon technology to accomplish unique and specific missions for disaster prevention, mitigation, and emergency response.

The aim of this paper is suggesting a complete wireless communications solution that can be deployed immediately, reliably and easily for emergency services and public safety. The attractive concept is the rapid deployment of Tethered Balloon that allows users to operate their existing mobile handsets. The main concern of this paper is to deliver broadband services with high quality of service during and after disaster. Then, rescue and relief teams will have the ability to perform their duty very effectively and efficiently in a catastrophe area.

The main concern of this paper is to analysis the performance of delivering wireless broadband services for large coverage area of WiMAX station which allocated in the payload of Tethered Balloon. WiMAX is considered to be allocated in the payload of Tethered Balloon because it supports Broadband wireless communication. Such Balloons will have the ability to be deployed within a short period of time and have the potential to provide Broadband Fixed Wireless (BFW) access services to a large number of users over a wide area. Thus, delay and throughput are measured and analysed by using OPNET Model 14.5

The rest of this paper organized Tethered Balloon technology related works describes in section II. Section III presents proposed architecture for emergency communication via Tethered Balloon. Implementation of proposed network 
architecture using OPNET, and results discussion are presented in section IV and $\mathrm{V}$, respectively. Finally, paper comes up with conclusion in section VI.

\section{Related Work Tethered Balloon Technology}

Tethered Balloon is the newest technology for wireless communication, which operates at troposphere layer for atmospheric research and subsequently. It is the balloon that tethered to ground by using ropes. The length of the ropes and their weight are limited and depended on the maximum balloon altitude. Ropes are used to maintain balloon stable against the wind rose which have been already used in Bandung (is the capital of West Java province in Indonesia) [11]. The number of ropes depends on the wind speed forecast and altitude target.

Zeppelin Company launched a craft in 2000 , followed by many projects which focused on design of Balloon for various applications and accurate operation in different altitudes [12]. Then, in 2003, CAPANINA project focused on providing broadband services [13]. Internet connectivity all over the world using balloon network has proposed by Google Project 'Loon' in 2011. Authors in [16] aimed to develop an airship for various applications such as surveillance, disaster management and advertisement. In [17] balloon established a long distance wireless communication link to provide Internet connectivity in rural areas. Researchers in [18] have attempted to provide telecommunication and Broadband services in remote and hilly areas.

Further, authors in [19] emphasized the importance of aerial platform during an emergency, which could significantly improve communications between rescue teams and headquarters, thus limiting human, environmental and economic consequences of the disaster. Due to HAP coverage, survivability and capability of being continuously on a station [14], manage the traffic [15] and easy to mitigate interference that occurs in the traditional wireless communication [16]. K. Mase has presented communication needs of evacuees in shelters during a post-disaster period. Those in shelters used to maintain communication channels with those outside shelters [17]. Therefore, aerial platform communication infrastructure is needed and it should be operated organically when all of the wireless communication is destroyed. The aerial platform should provide E911 location capability to facilitate search and rescue operations [18]. Rescue and relief teams should be given high priority and reserved channels in an emergency areas and situations [19]. 
. ABSOLUTE has aimed to implement LTE-A aerial base stations using LAP to cover and provide capacity for public safety usage in the aftermath and during the disasters [21]. On the other hand, CAPANINA project has steerable antenna for delivering broadband wireless services to the users under the coverage of HAP [22].

Presently, Tethered Balloon technology has drawn attention of researchers and has considered as new technology for broadband wireless communication. Many programs and researches from all over the globe are pursuing the design and development of various applications that are based on this technology. It has the potential to deliver wide variety of telecommunication services by overcoming various challenges faced in traditional cellular systems such as limited coverage area, high power consumption, delay, high power consumption, lack of line of sight and etc.

The integration of satellite, LAP and terrestrial network was proposed for public safety which can deliver services to wide coverage area, and provide higher throughput [28].

\section{Tethered Balloon Technology}

Space technologies have recently become popular as key technology for deliver broadband wireless communication. Aerial platforms represent the key enablers for rapid deployable wireless communication and capable of delivering broadband wireless communication for emergency services and public safety agencies in any disaster event. Arial platform categories are represented as High Altitude Platforms (HAP) which placed in stratosphere layer and operated at an altitude of 17-22 Km [9], Medium Altitude Platform (MAP) and Low Altitude Platform (LAP) which placed in placed in troposphere layer $[9,20]$. The comparison between space technologies is shown in Table.1. The competitive system has attracted the attention, offering solutions for the bottlenecks with the satellite system such as aerial platforms. Aerial platforms are capable of providing broadband wireless communication and remote sensing. The most important features of aerial platforms are: lower cost of installation and maintenance, low delay time, and etc. $[9,20]$.

Tethered Balloon is one of LAP families which operates at altitude 200- 440 $\mathrm{m}$ [9]. The coverage provided via Tethered Balloon is $5.5 \mathrm{Km}$ radius while the altitude of balloon $440 \mathrm{~m}$ [11]. The coverage can be extended with increasing 
balloon altitude or increasing the number of balloons []. By increasing the number of Tethered Balloon, balloons can communicate with each other using fiber for high-speed data transfer. For society, Tethered Balloon able to mitigate the health risk due to exposure to radiation and environmental effects and reduce the carbon footprint in atmosphere. Furthermore, Tethered Balloon has the ability to bring-up communication infrastructure quickly, during disastrous situations and green field remote area [11].

Table. 1 comparison of satellite and Tethered Balloon and terrestrial systems

\begin{tabular}{|l|l|l|}
\hline \multirow{2}{*}{ Terrestrial } & Advantages & Disadavntages \\
\hline \multirow{2}{*}{ satellite } & Various multimedia sevices & vulnerable to disaster \\
\cline { 2 - 3 } & Capacity enhanced & \\
\hline \multirow{2}{*}{ Tethered Balloon } & Large coverage area & High delay \\
\cline { 2 - 3 } & Less effected by diaster & Require LoS \\
\hline & LoS & Small coverage area \\
\cline { 2 - 3 } & Neglactable delay & $\begin{array}{l}\text { Not aplicable for all type of } \\
\text { disasters }\end{array}$ \\
\hline & Easy and fast deplyment & \\
\hline & Less effected by disaster & \\
\hline
\end{tabular}

\section{Tethered Balloon Network Architecture}

Tethered Balloon is used for provide wide coverage area, in order to demonstrate the effectiveness of broadband communication network. The network architecture of Tethered Balloon system consist of sky segments, ground segments and tethered as shown in Fig.

The ground segments include the user equipment, main unit of base station and power system. The user equipment represents the terminal users which should support full mobility during user operations. However, the base station main unit connects with the transceiver and interface to the Power Supply Unit (PSU) - 48V DC as well as connect the network with others existing networks. Single MU provides synchronization between network core and the base station. The ground station acts as a hub for the network and provides service to customers who are in direct LoS with tethered balloon antenna and within the cell radius. 


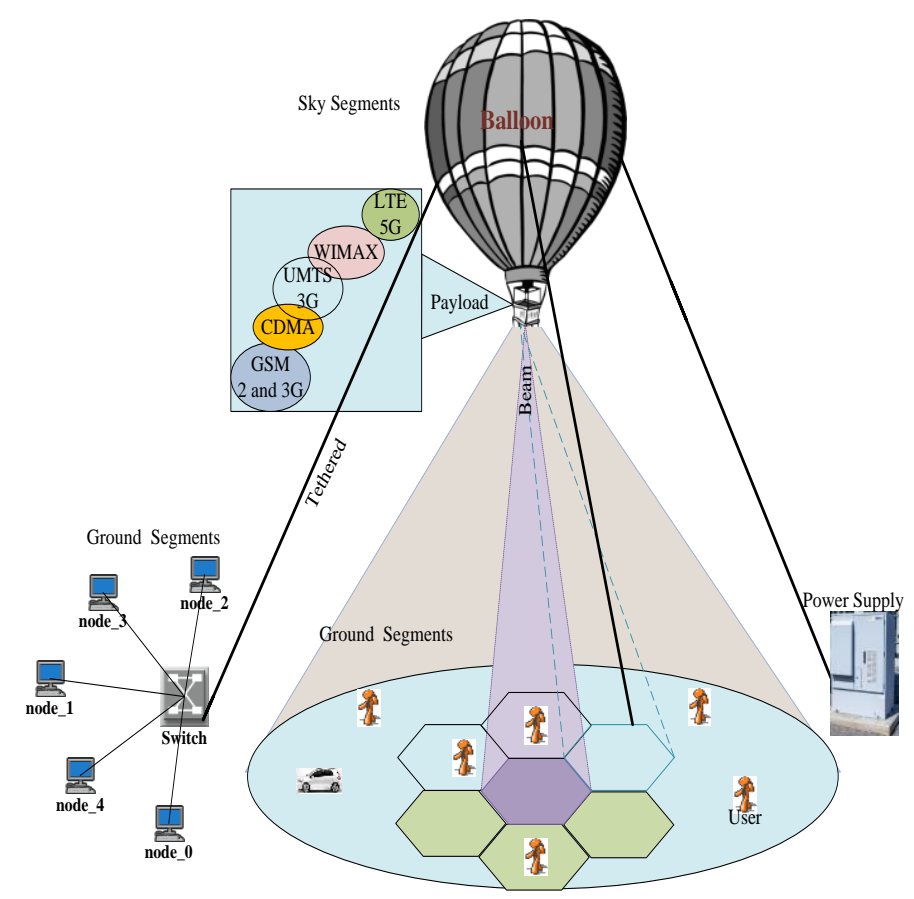

Fig.2. Tethered Balloon network Architecture

The space segment connects the mobile terminal with the central unit. It consists of balloon, payload. The payload comprises of Radio Remote Unit (RRU) and antennas for mobile wireless communications. Tethered Balloon Payload can be implemented with various wireless technologies such as: $3 \mathrm{G}$, Wi-Fi, WiMAX, LTE and LTE-A or the combination of more than one [10, 24, 25]. In case of using multiballoon, the connection link between them is fiber for high-speed data transfer.

Tether is the connection link between balloon and other networks in the ground. Also, Balloon is restrained by tether to maintain the balloon stable in position. These tethers can also provide power for the payload from the ground [26]. It is assumed to be of circular section, sufficiently rough to ensure turbulent wakes at limited wind speed of $30 \mathrm{~m} / \mathrm{s}$, giving a drag coefficient of 0.3 [26]. Tethered varies with altitude, because tethered is assumed to be supported by balloon as well as the tethered winch is fixed to the ground. Furthermore, It provides a connection between Remote Radio Unit (RRU) and MU to transmit power and data. The advantages of using tethered are:

1) Minimized the need for propulsion to keep balloon in high winds.

2) Send communication signals and video feed.

3) Used for supply payload power in case of not using solar power.

4) Provide low-pressure waveguides to convey millimeter wave power.

There are many advantages of using aerial platforms in such as earthquakes, tsunamis, etc. 


\section{A. Single Tethered Balloon Scenario}

A single Tethered Balloon is envisaged for providing a communication network services for special events such as disaster recovery, rapid broadband employment over highly populated citied as shown in Fig. 2. Tethered can keep Tethered Balloon the quasi-stationary to the ground. Tethered balloon can be used as a stand-alone for providing coverage to disaster area and groups of rescue and relief teams.

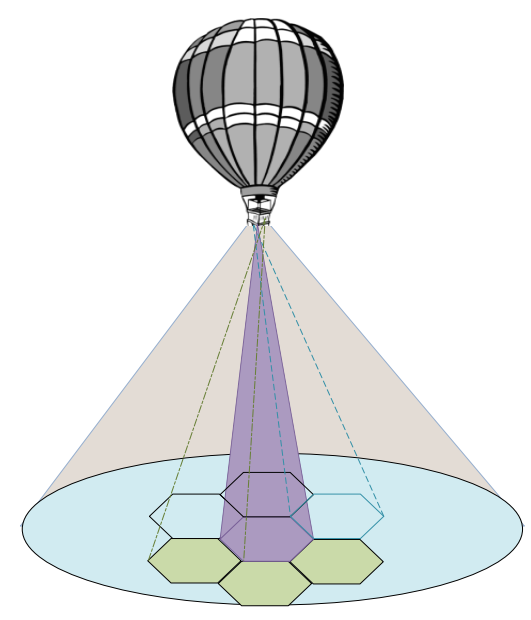

Fig. 2 Single tethered balloon

\section{B. Multi-Tethered Balloon}

The idea of increasing the number of Tethered Balloons serving a common coverage area is to increase the capacity and the bandwidth efficiency. Furthermore, Multi-Tethered Balloon constellations can increase the capacity by exploiting the directionality of the fixed user antenna. The reason for balloon configuration is to provide resilience and to provide unique diversity to end users receiving antennas for improved service availability. In the case of multi-Tethered Balloon constellations, they can be interconnected via ground stations or inter-balloon link (IBL) between two balloons represents an additional network interface. IBLs can be radio or optical, thus arbitrarily extending the system coverage. In fact, the coverage area is spilt into multiple cells to increasing the capacity. In addition, Multi-Tethered Balloon constellations could enhance the overall system capacity in overlapping coverage. A general Tethered Balloon constellations architecture is depicted in Fig. 3. 


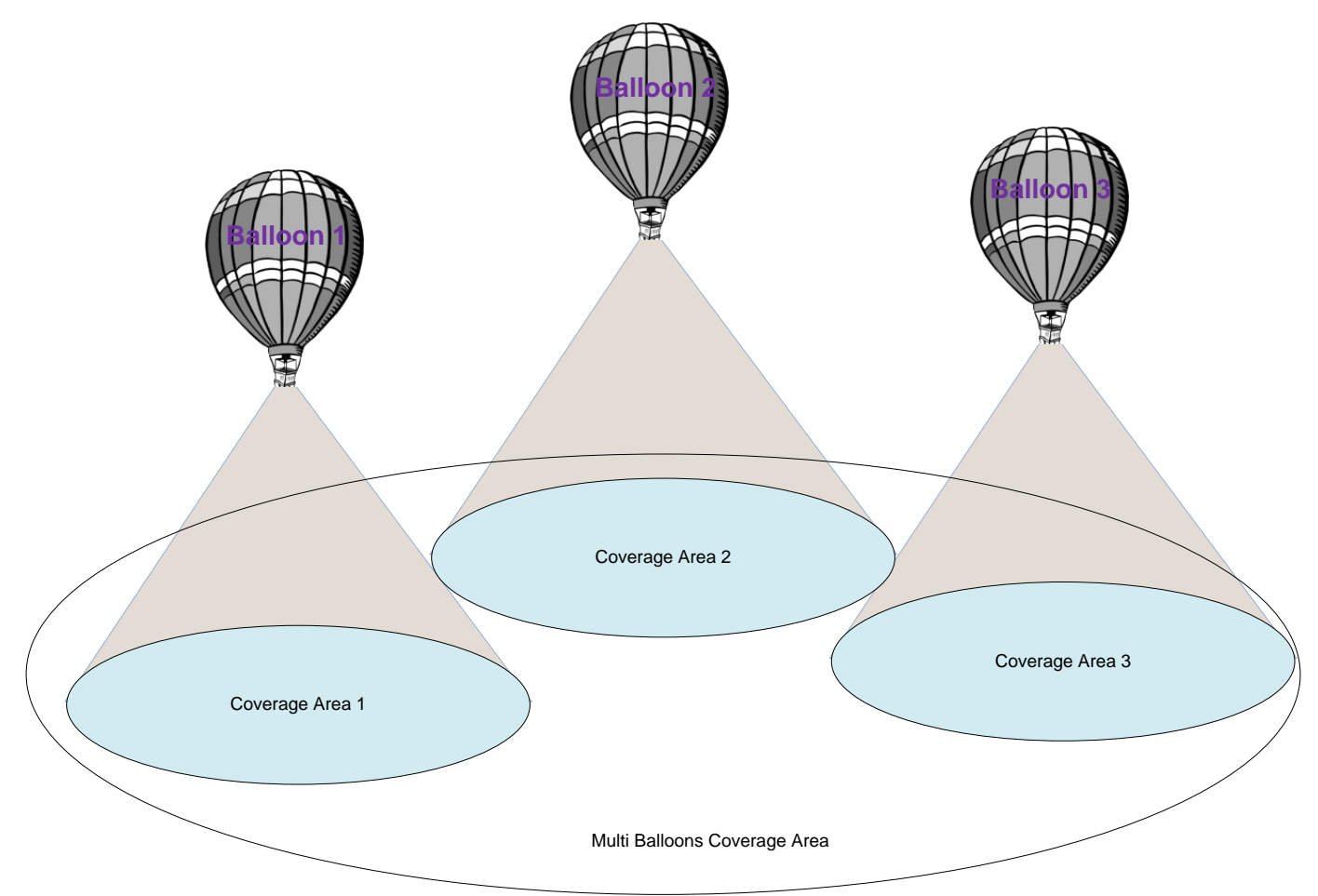

Fig. 3 Multiple Tethered Balloon

\section{Tethered Balloon for public safety networks}

Wireless communication network infrastructure can be damaged due to network component physical destruction, during the unexpected and natural hazardous. The disaster can heavily affect the terrestrial wireless network architecture performance or can fully paralyze it. Therefore, aerial platforms are intended to deliver provision of broadband services to large coverage area [27]. Tethered Balloon was chosen instead of others aerial platforms because of the advantages it offers such as rapid deployment and lower cost.

Emergency communication is one of the most important specialized fields. It applies to provide and maintain, operable and interoperable wireless services and the internet before, during and after presidentially declared emergencies anywhere [29]. Therefore, rescue and relief team operations totally depend on reliable, efficient and fast emergency communication system [30]. The highest priority is given to provide an emergency communication services i.e. more valuable than general communication services. Therefore, the requirements for space communication technology potentially attracted for designing new network architecture to recovery and manage disasters [31]. The main components of emergency communications are 911 call processing and delivery through public safety authorities [32]. These components have to operate efficiently to achieve a satisfactory response, which consist of three primary elements: 
I. Operability: it is the ability of emergency responders to establish and sustain connections.

II. Interoperability: it is the capability of emergency responders to communicate among jurisdictions, disciplines, using a variety of frequency bands.

III. Continuity: it is the ability of emergency response agencies to maintain communications in the event of destroys.

Tethered Balloon lifts a flying platform with incorporating WiMAX equipment to provide efficient communication services. Particular in this network, relief and rescue team also use WiMAX coverage from Tethered Balloon to get Internet, VoIP and Video Conference services. The most important benefits of using Tethered Balloon for emergency communication are easy to deploy, easy to maintains and low cost of lunching. At what time of Tethered Balloon reaches the desired altitude, the video camera will capture the affected area. Then, it keeps flying incorporating WiMAX in the height of emergency area.

Capture images will be sent to emergency headquarters for analyzing the emergency area. This helps emergency headquarters to coordinate search and rescue teams, with an arrival of first responders. In this way, Tethered Balloon provides vital role to offer the critical path for rescue and relief teams in emergency area. Hence, the importance of balloon for delivering emergency can be summaries as:
A. To guide rescuers and reliefs team
B. Deliver rescue tools, sensors and medical supplies.
C. Provide mobile telephone connectivity to coordinate search, rescue and logistics.
D. Deliver power. 
The proposed emergency Tethered Balloon network architecture is shown in Fig.

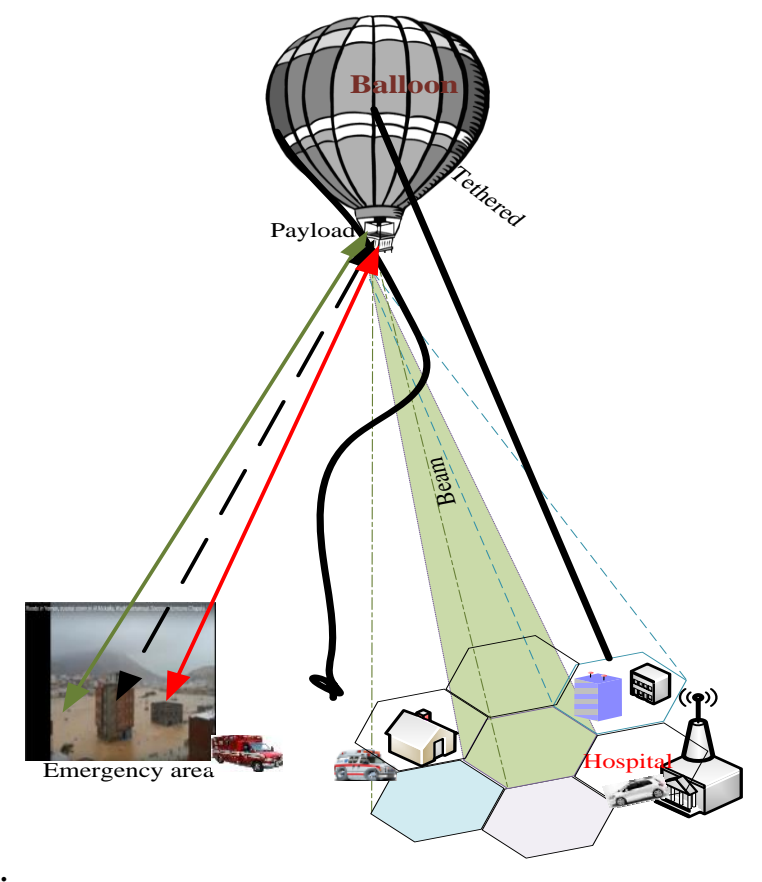

Fig.3 Emergency Network Architecture

Fig.3 Emergency Network Architecture

Furthermore, concern of this network is also to incorporate all communication capabilities in Tethered Balloon. Therefore, it could interoperate with all existing networks. Tethered Balloon cellular should be configured for special access profiles without paying attention to wireless communication technology.

Recently, the Tethered Balloon technology represents the most powerful and promising technology to improve public safety network and emergency operations for saving people lives during and after disaster. Tethered Balloon with help of WiMAX technology in the payload is used for deliver broadband services to the disaster area. Therefore, people will enjoy high quality of service and strong signal in anywhere under Tethered balloon coverage area.

The public safety network plays a vital role on protect the citizens, lifesaving and other activities; disaster, accident and terrorist attacks. The team coordination can be enhanced effectively and efficiently via reliable implementation of broadband service for different devices under the coverage of Tethered Balloon and contacting the headquarters. Moreover, the public safety networks need to cater the demand to high quality of services for ensuring that the coordination activities during the aftermath of a disaster are effective. Therefore, Tethered balloon is an economic choice 
and more feasible for public safety broadband network deployment where any event occurred. Due to Line of sight, Tethered balloon can cover large areas and very low delay which can be neglectable.

Tethered Balloon Technology can be used as stand-alone system for providing coverage to the group terminals to connect the first response to headquarters.

Tethered balloon dedicated plays an essential role for public safety network communication in the field first response. The infrastructure of Tethered Balloon based public safety network consists of Balloon which carries the wireless communication station and Vehicle, rescue and releif teams and cener control and analysis unit. Using such equipments brings many benfit to increase the coverage area, enhanceing the capacity, and improving the propabaility of success network. Herefore, the utilization of Tethered Balloon is considered as an alternative to the destoryed wireless communication technologies and become an esential for public safety.

The proposed network comprises two parts which are Balloon and the survived terrestrial network. The Tethered Balloon is very easy to deploy and maintain. Therefore, immediately can be fast deploy and replace the destroyed communication technologies.

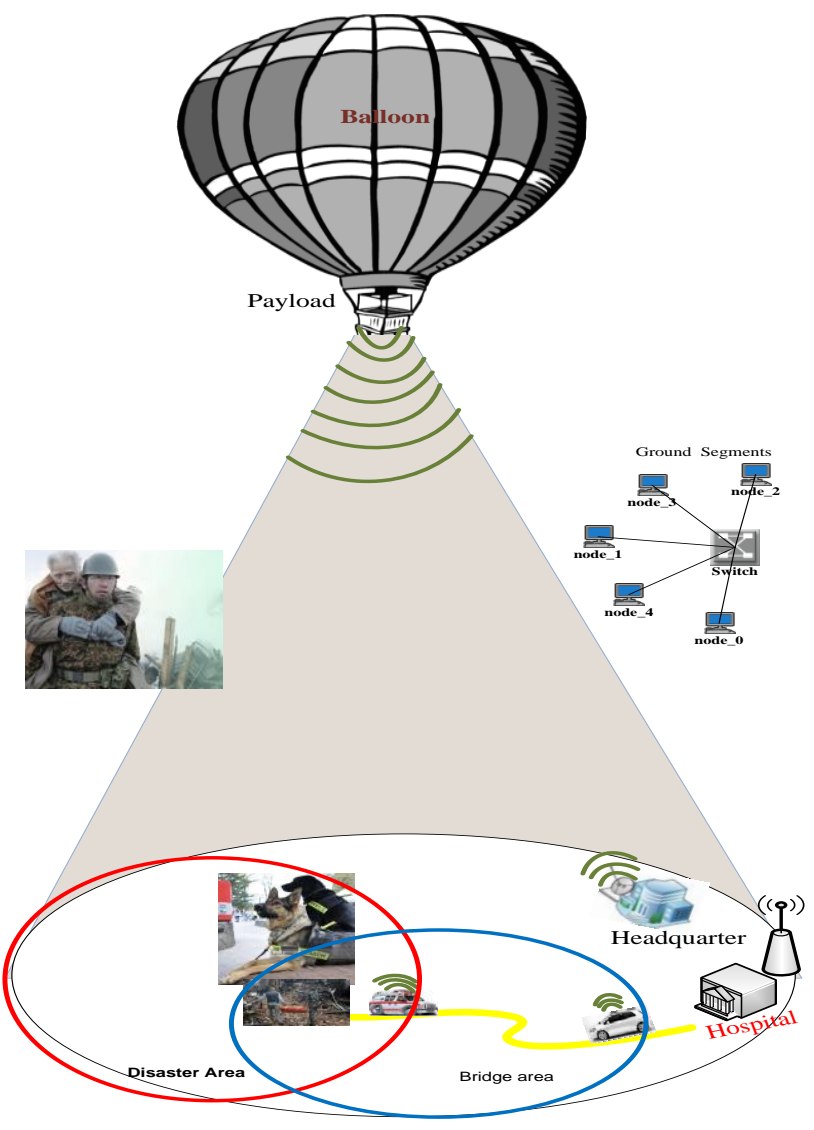

Fig. Public Safety and Emergency communication

Public safety network is used to respond and prevent of the disaster harm for endanger persons. 
Using the required component for emergency and disaster recovery can demonstrate the rescue and relief teams and will efficiently search for people in catastrophic area.

\section{Implementation of Proposed Network by using OPNET}

Designing an efficient network plays an important role in this world. Even, it is an essential part to check the performance of the designed network, which will be a difficult task in real time applications. For this network, OPNET Modeler considers one of the most reputed simulators that have been designed so far. OPNET Modeler provides different levels of modeling depending on the necessities and requirements of simulation. The operator of this model can develop several network scenarios in order to evaluate and analyze network performance. The design of this particular network contains of Tethered Balloon that carries WiMAX Base Station (WBS) and five nodes represented rescue and relief teams.

Our scenario focuses on the performance of rescue and relief teams in order to provide rescue and relief team high priority to deliver broadband service. Therefore, they are able to perform their duty in best scenario possible along with providing the necessary medicine to save people life. The scenario consists of three teams as shown in Fig.4. Where node- 1 and node-4 represent the first team that works in the emergency area for relief operations (rescue teams), in order to bridge people during to the safe area. Node- 3 , node- 0 and node 2 represent the second team that their duty is to move the evacuees from edge of emergency area to hospital in order to save their life. Third team works in emergency headquarters office. Their duty is to monitoring the emergency area, analysis the capture image and communicates with the rescue team to direct and guide them. Here, Tethered Balloon is used to provide needed critical communication for search and rescue as well as control and command as shown in Fig.4. 

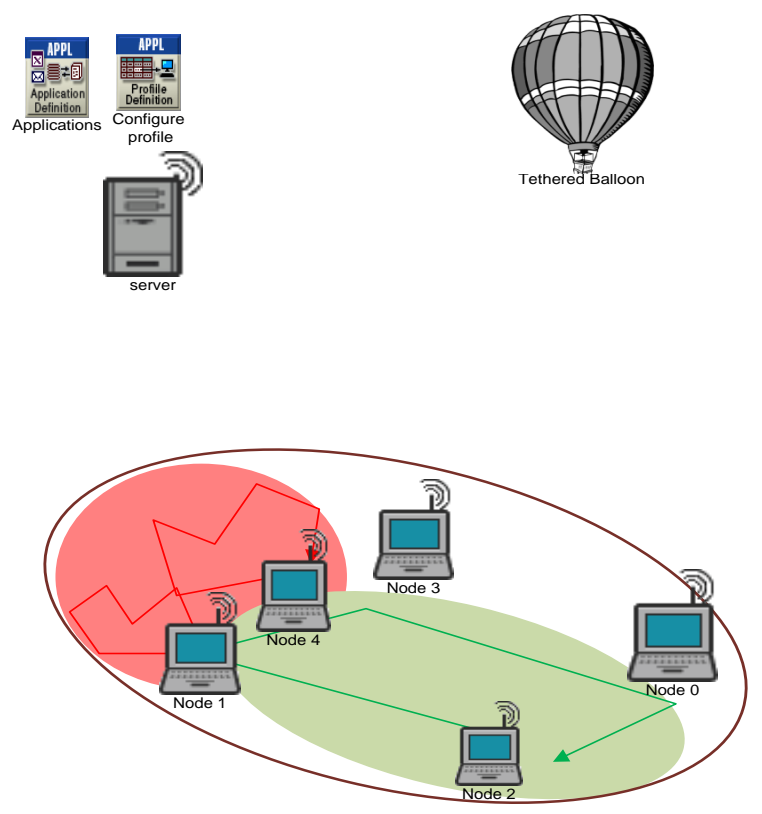

Fig.4. Impiment proposed network using OPNET

\section{Analysis the performance}

The network performance evaluation needs different kinds of parameters. Delay and throughput parameters are used to test the network performance evaluation. In a communication network, delay and throughput are very important to be considered when evaluate network performance required.

\subsection{Delay}

Delay specifies how long it takes for a bit of data to travel across the network from one user to another. It is typically measure in fractions of second. The packet end-to-end delay is the time of generation of a packet by the source up to the destination reception. This time is expressed in sec. Hence, all the delays in the network are called packet end-to-end delay. There are different kinds of activities because of which network delay is increased. We have several kinds of delays such as processing delay $D_{p}$, transmission delay $D_{t}$ and propagation delay $D_{d}$. Suppose if there are $N$ number of nodes, then the total delay can be calculated by taking the average of all the packets, source destination pairs and network configuration.

$$
\mathrm{D}_{\mathrm{e}-\mathrm{e}}=\mathrm{N}\left(\mathrm{D}_{\mathrm{t}}+\mathrm{D}_{\mathrm{d}}+\mathrm{D}_{\mathrm{p}}\right)
$$

Where $D_{e-e}$ is end delay and $D_{t}$ is time it takes to push the packet's bit on link.

\subsection{Throughput Analysis}

Throughput is the number of data successfully delivered per unit time. It is controlled by available bandwidth, signal to noise ratio. Throughput is to be measure from the arrival of the first bit of data at the receiver. System throughput is the sum of the data rates that are delivered to all terminals in a network. System throughput is the fraction of time which channel is used for transmit payload bits successfully. There are three random chosen slot corresponding's such as an idle slot, a collision, and a successful transmission which represented by probability of idle slot $\left(P_{\text {idel }}\right.$, probability of collision $\left(P_{c o l}\right)$ and probability of successful transmission $\left(P_{s u c}\right)$, respectively. In addition, that probability occurred during particular time called idle slot time, collision time $T_{c o l}$ and successful transmission $T_{s u c}$, respectively. The average of duration time is represented by $T_{\text {avr }}$ and is given by equation (7).

$$
T_{\text {avr }}=P_{\text {idel }} \delta+P_{\text {succ }} T_{\text {succ }}+P_{\text {col }} T_{\text {col }} \ldots \ldots \ldots \ldots
$$

On the other hand, throughput is represented by $S$ and calculated by using equation (8).

$$
S=P_{\text {succ }} E[p] / T_{\text {avr }}
$$

Where, $E[p]$ is Payload information transmitted in a slot time which is average payload size. 
$=P_{\text {succ }} E[p] / P_{\text {idel }} \delta+P_{\text {succ }} T_{\text {succ }}+P_{\text {col }} T_{\text {col }}$

By dividing the numerator and denominator of equation (3) by $\left(P_{\text {succ }} T_{\text {succ }}\right)$, the throughput can be expressed as follows:

$$
\begin{aligned}
S=E[p] / T_{\text {suc }} & {\left[1+P_{\text {col }} / P_{\text {suc }}+T_{\text {col }} / T_{\text {suc }}\right.} \\
+ & \left.P_{\text {idel }} / P_{\text {suc }}+\delta / T_{\text {suc }}\right]
\end{aligned}
$$

Accordingly, for compute the throughput for a given handshake, we only need to specify the corresponding values of $T_{c o l}$ and $T_{\text {suc }}$.

\section{Results and discussions}

The results show the importance of using Tethered Balloon as new technology for emergency communication using WiMAX payloads that can support broadband wireless access. Therefore, the communication facilities (calls and Internet) during emergency situation improve excellently.

Fig. 5 shows Jitter decreases from $0.000232 \mathrm{Sec}$ to be nearest to zero when the time increases. Negative Jitter indicates that the time difference between the packets at the destination node was less than the source node. End to end delay for a voice packet is measured from the created time to the received time. Here, end to end delay varies from minimum $0.1282 \mathrm{Sec}$ to maximum $0.200 \mathrm{Sec}$.

Traffic voice received 2194.66 to 47744.4. Traffic sent 3444.67 to 79543.5 . Average number of bytes per second submitted to the transport layers by all voice applications in the network. While time increases the Packet delay variation increases till maximum value 0.01722. Average number of bytes per second forwarded to all voice applications by the transport layers in the network. 


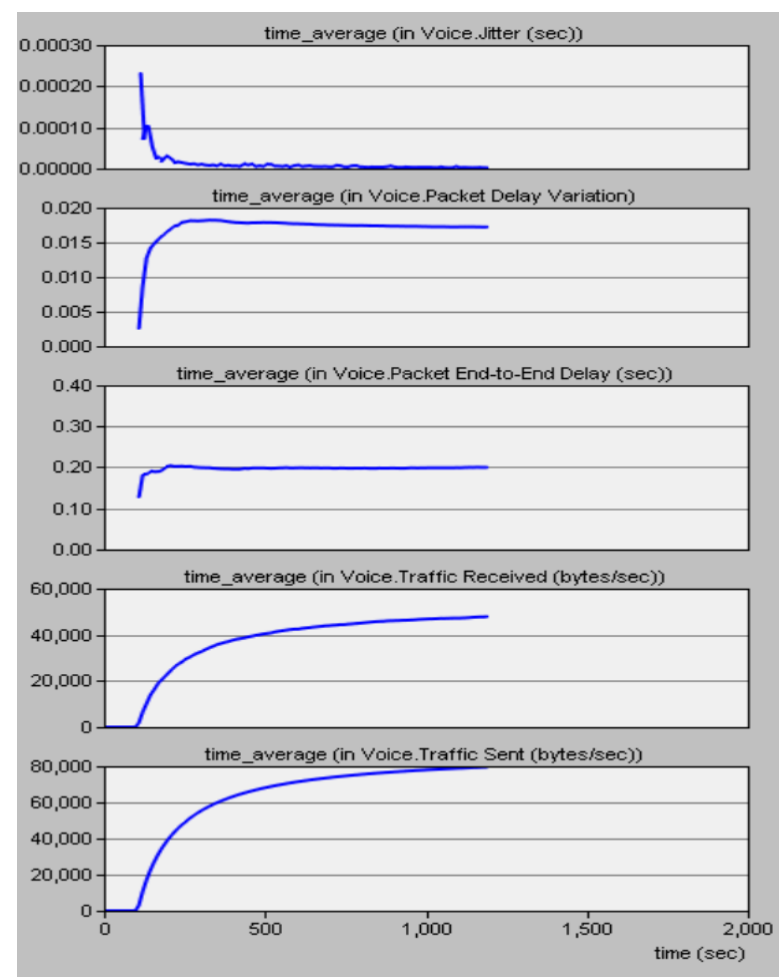

Fig.5. Voice Network Characteristics

Delay of WiMAX in Tethered Balloon is from 0.003845 to $0.074777 \mathrm{Sec}$ as shown in Fig.6. It represents the end to end delay of all packets received from Tethered Balloon MACs of all WiMAX nodes forwarded to the higher layer. Load represents the total load (in bits/Sec) submitted to WiMAX layers in all WiMAX nodes of the network. Load is 34.6667 to 3442285.893 as shown in Fig.6. Throughput represents the total data traffic (in bits/Sec) forwarded from WiMAX lower layers to higher layers in all WiMAX nodes. Throughput is increased from 208 to $2420475.653 \mathrm{bits} / \mathrm{Sec}$.

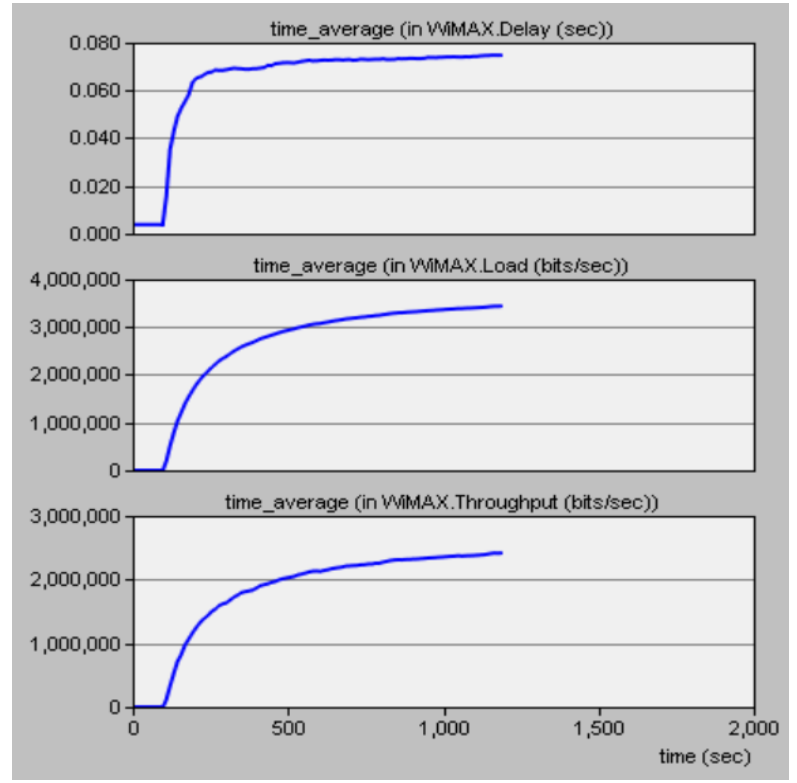

Fig.6. Network characteristics 
Rescue teams are represented by node- 1 to node- 4 as shown in Fig.4. Node1 and node- 4 are shown in the emergency area; they are guided by people who are analyzed the image that capture from Tethered Balloon for particular area. Therefore, the traffic received by node- 1 and node- 4 is better than others because it given higher priority than others as shown in Fig.7.

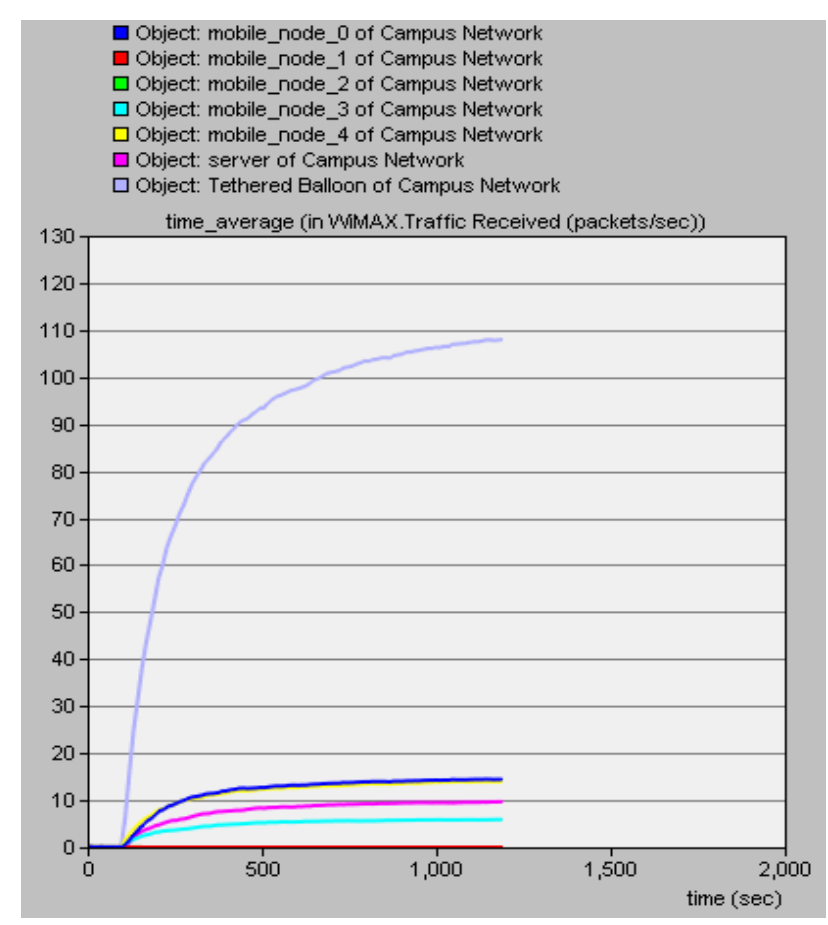

Fig.7. Scenario Traffic Received

Delay is affected either by increasing number of mobile nodes or increasing the packet size. Delay was constant at $0.00384 \mathrm{Sec}$ with time varies from 0 to 84 Sec as shown in Fig.8. For node 3, delay of setup increases between 84 to $156 \mathrm{Sec}$, and it decreases between 0.01375 to $0.01080 \mathrm{Sec}$, as the time change between 168 to $1188 \mathrm{Sec}$. The server decreases from 108 to 1188 , when delay decrease from 0.00450 to $0.00200 \mathrm{Sec}$. 


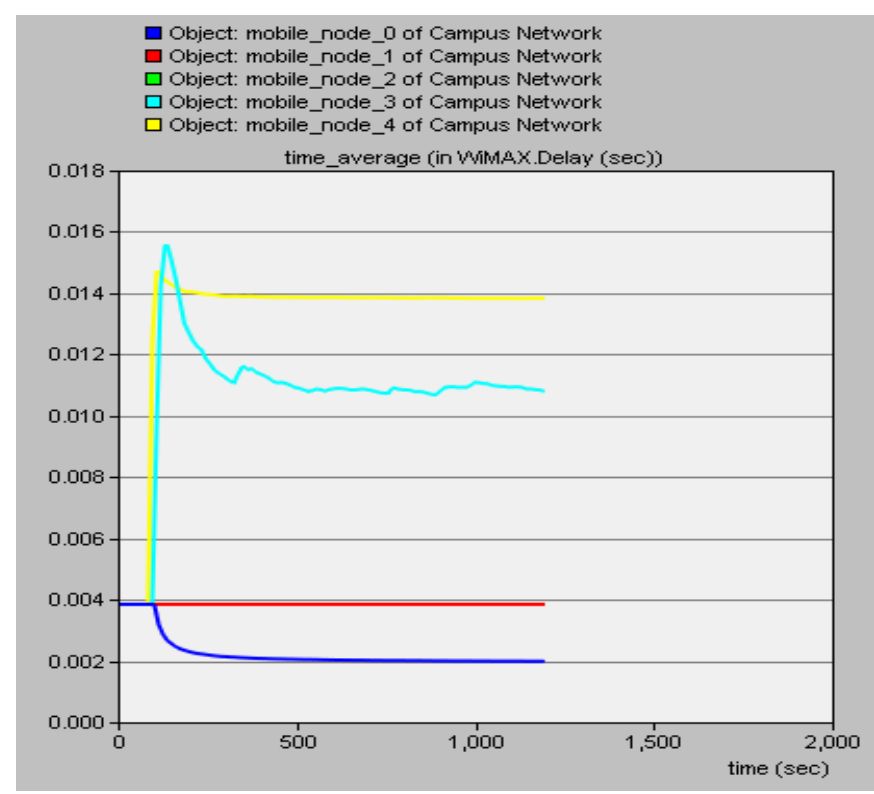

Fig.8. Different delay for different nodes

Fig.9 shows the server characteristics such as: load, traffic received and sent. Load of proposed network significantly increase because of emergency situation. The traffic received in Tethered Balloon is higher than traffic sent; because all mobile users send traffic request and make call in that particular area.

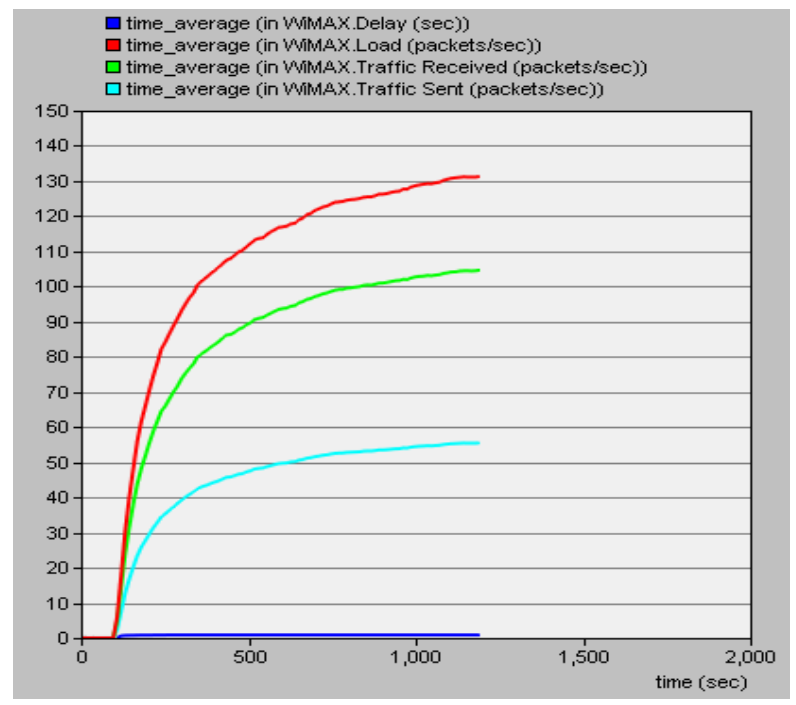

Fig.9. Server characteristics and parameters

In wireless communication, SNR is a significant parameter that influences the system performance. Fig.10 demonstrates the value of SNR in both cases downlink and uplink. Downlink SNR value represents packet transmissions from WiMAX via Tethered Balloon. For SS nodes, the SNR measured at particular SS for all packets arriving from WiMAX BS in payload of Tethered Balloon. Uplink represents the packet transmissions through the WiMAX. From Fig. 10, it is clear that the SNR value of downlink shows better performance than the uplink. It may 
be due to the lower User Equipment (UE) transmission power in comparison to the Balloon transmission power.

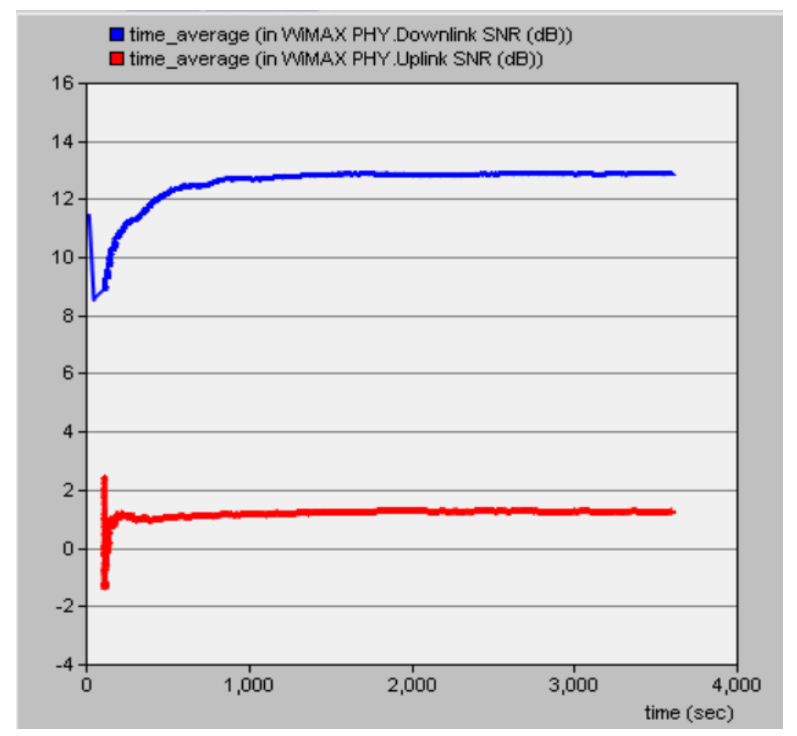

Fig. 10 Uplink and Downlink SNR

The call admission control (CAC) is implemented in the BS [15] and in our particular scenario, CAC is implemented in the WiMAX BS that allocated in the Tethered Balloon payload. Fig.11 demonstrates the total capacity of uplink and down link as well as number of admitted and rejected calls. In this particular scenario, the number of admitted calls is 30 and rejected calls is zero. It can also be observed that downlink capacity (throughput) is greater than uplink because of high demand from end user devices on emergency area.

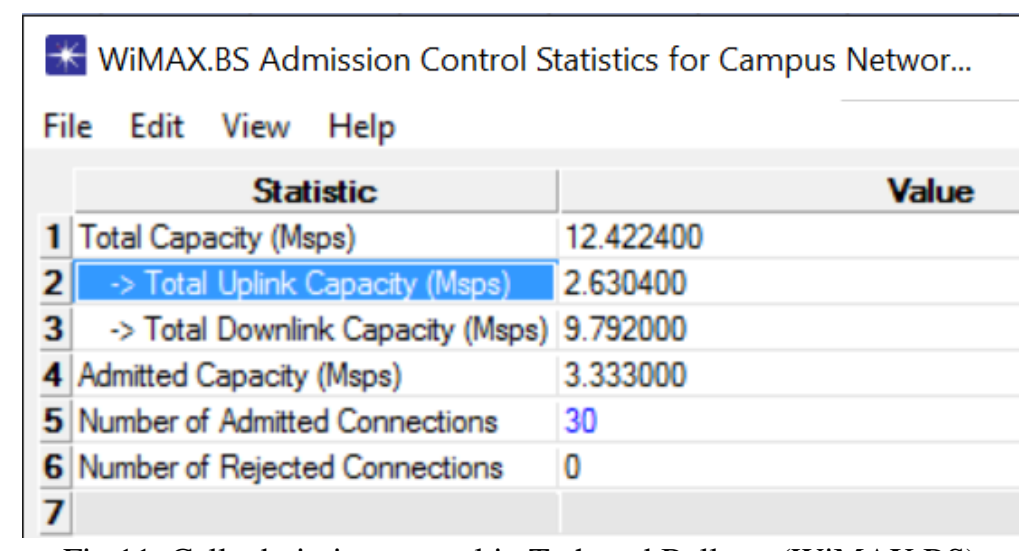

Fig.11. Call admission control in Tethered Balloon (WiMAX BS)

Per

This scenario is considered and implemented using OPNET 14.5. Delay and throughput are considered to measure and evaluate the performance of proposed network.

Fig.7. is shown that delay is increase from 0.0039 to 0.0045 with increasing time significantly. Also the load and throughput are increased significantly. Load is increased from 1047.7 to 859117.76 and throughput also increases from 1031.1 to 859089.6. 
Delay of different user is different that because of the different environments and different position from the center of tethered Balloon as shown in Fig.8. Tethered Balloon delay is increase but still very less from 0.0029 to $0.00337 \mathrm{Sec}$. on the other hand delay of mobile user nodes is different according to the position of user and the distance of user from the center coverage area of Tethered balloon as shown in Fig.8.

Throughput in tethered balloon is very high, but it is low in the mobile users. Throughput is increased with time as shown in Fig.9.

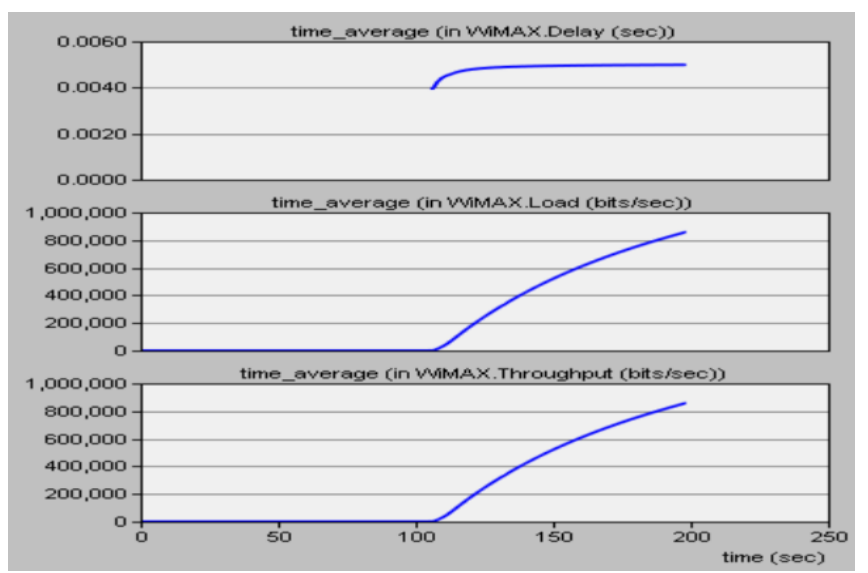

Fig.7. Network delay

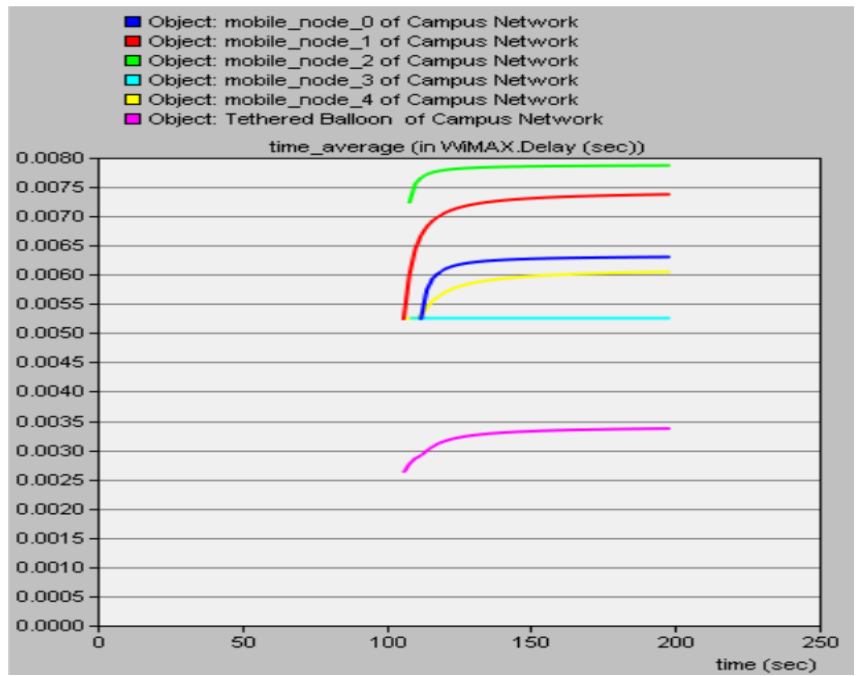

Fig.8. Delay

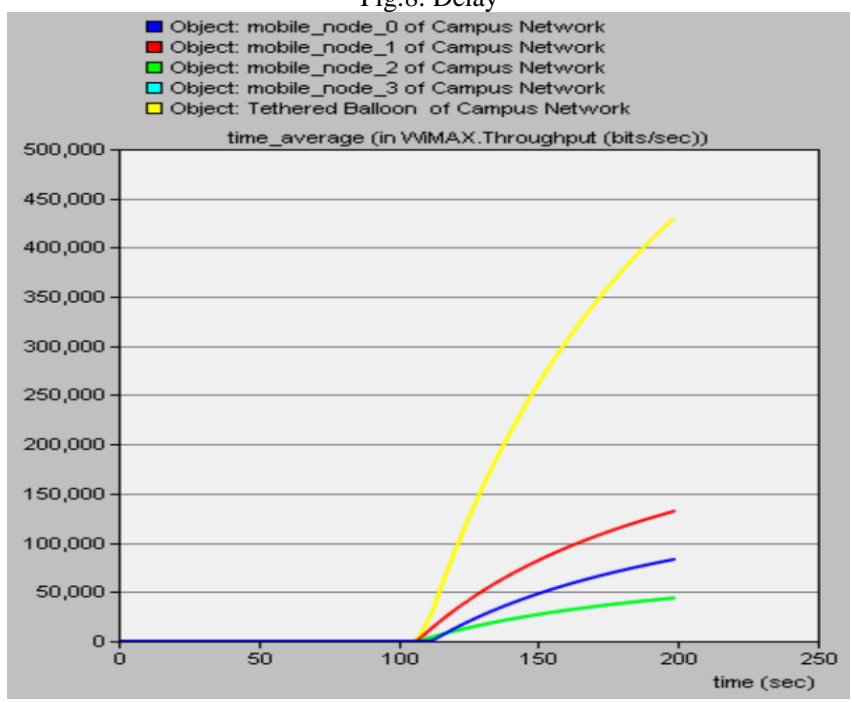

Fig.9.Throughput 


\section{Conclusion}

In this paper, we have proposed wireless broadband delivery from Tethered Balloon. Tethered Balloon is balloon tethered to ground and operate at $200 \mathrm{~m}$ above ground. Then, analysis and simulation of the performance of proposed network is done by using OPNET Model 14.5. Delay and throughput are considered evaluate the performance of particular network. The results shown the significant effectiveness of using WiMAX BS in the payload of Tethered Balloon for delivering broadband services in rural and remote areas.

\section{Conclusion}

Wireless Communication network is foundation and key of the development of emergency communication using space-based technologies such as aerial platforms. This paper describes importance of Tethered Balloon as a communication technology for emergency communication. Rescue and relief teams are given high priority to perform their duty in good manner. Understanding the traffic emergency communication is very difficult due to big data that require for estimation. Therefore, this study focuses on the analyses and simulates the performance of the relief and rescue teams in emergency area using OPNET Model. Here, voice network characteristics, traffic received, delay, SNR, throughput and CAC are taken into account in order to observe the network performance. It is also noticed that the Tethered Balloon provides a great alternative solution for emergency communications and assists in saving lives.

\section{References}

[1] D. Komnakos, D. Vouyioukas, I. Maglogiannis, and P. Constantinou, "Performance evaluation of an enhanced uplink 3.5 G system for mobile healthcare applications," International journal of telemedicine and applications, vol. 2008, 2008.

[2] Y.-M. Lee, B.-1. Ku, and D.-S. Ahn, "A satellite core network system for emergency management and disaster recovery," in Information and Communication Technology Convergence (ICTC), 2010 International Conference on, 2010, pp. 549-552.

[3] J. Yoo, "Performance Evaluation of Voice Over IP on WiMAX and Wi-Fi Based Networks," Communication Networks 2009.

[4] J. D. Deaton, "High Altitude Platforms for Disaster Recovery:Capabilities, Strategies, and Techniques for EmergencyTelecommunications.," EURASIP Journal on Wireless Communications and Networking vol. 2008, pp. 1-8, 2008.

[5] B. T. Ahmed and M. C. Ramon, "WiMAX in High Altitude Platforms (HAPs) Communications over Large Cities," in 6th International MultiConference on Systems, Signals and Devices, 2009, 2009, pp. 1-4.

[6] P. Likitthanasate, D. Grace, and P. D. Mitchell, "Coexistence Performance of High Altitude Platform and Terrestrial Systems Sharing a Common 
Downlink WiMAX Frequency Band," Electronics Letters, vol. 41, pp. 858860, 2005.

[7] S. H. Alsamhi and N. S. Rajput, "Neural Network in A Joint HAPS and Terrestrial Fixed Broadband System," International Journal of Technological Exploration and Learning (IJTEL), vol. 3, pp. 344-348, 2014.

[8] S. Khaleefa, S. Alsamhi, and N. Rajput, "Tethered balloon technology for telecommunication, coverage and path loss," in Electrical, Electronics and Computer Science (SCEECS), 2014 IEEE Students' Conference on, 2014, pp. 1-4.

[9] S. H. Alsamhi and N. S. Rajput, "An Intelligent HAP for Broadband Wireless Communications: Developments, QoS and Applications," International Journal of Electronics and Electrical Engineering, vol. 3, 2015.

[10] A. Qiantori, A. B. Sutiono, H. Hariyanto, H. Suwa, and T. Ohta, "An emergency medical communications system by low altitude platform at the early stages of a natural disaster in Indonesia," Journal of medical systems, vol. 36, pp. 41-52, 2012.

[11] H. Hariyanto, H. Santoso, and A. K. Widiawan, "Emergency broadband access network using low altitude platform," in Instrumentation, Communications, Information Technology, and Biomedical Engineering (ICICI-BME), 2009 International Conference on, 2009, pp. 1-6.

[12] T. C. Tozer and D. Grace, "High-altitude platforms for wireless communications," Electronics \& Communication Engineering Journal, vol. 13, pp. 127-137, 2001.

[13] D. Grace, M. Mohorcic, M. Oodo, M. Capstick, M. B. Pallavicini, and M. Lalovic, "CAPANINA - communications from aerial platform networks delivering broadband information for all," Proceedings of the 14th IST Mobile and Wireless and Communications Summit, 2005.

[14] S. H. Alsamhi and N. S. Rajput, "An Intelligent Hand-off Algorithm to Enhance Quality of Service in High Altitude Platforms Using Neural Network," Wireless Personal Communications, vol. 82, pp. 2059-2073, 2015/06/01 2015.

[15] S. H. Alsamhi and N. S. Rajput, "Implementation of call admission control technique in HAP for enhanced QoS in wireless network deployment," Telecommunication Systems, pp. 1-11, 2015.

[16] S. H. Alsamhi and N. S. Rajput, "Methodology for Mitigation of Interferences from High Altitude Platform Ground Station to Terrestrial Stations," international journal of Scientific \& Engineering Research (IJSER), vol. 3, pp. 1-7, 2012.

[17] K. Mase, "How to deliver your message from/to a disaster area," IEEE Communications Magazine, vol. 49, pp. 52-57, 2011.

[18] A. Mohammed, S. Arnon, D. Grace, M. Mondin, and R. Miura, "Advanced Communication Techniques and Applications for High-Altitude Platforms," EURASIP Journal on Wireless Communications and Networking, vol. 2008, 2008.

[19] S. H. Alsamhi and N. S. Rajput, "An Efficient Channel Reservation Technique for Improved QoS for Mobile Communication Deployment Using High Altitude Platform," Wireless Personal Communications, pp. 114, 2016. 
[20] S. A. Khaleefa, S. H. Alsamhi, and N. S. Rajput, "Tethered balloon technology for telecommunication, coverage and path loss," in Electrical, Electronics and Computer Science (SCEECS), 2014 IEEE Students' Conference on, 2014, pp. 1-4.

[21] I. Bucaille, S. Hethuin, T. Rasheed, A. Munari, R. Hermenier, and S. Allsopp, "Rapidly deployable network for tactical applications: Aerial base station with opportunistic links for unattended and temporary events absolute example," in Military Communications Conference, MILCOM 2013-2013 IEEE, 2013, pp. 1116-1120.

[22] D. Grace, M. H. Capstick, M. Mohorcic, J. Horwath, M. B. Pallavicini, and M. Fitch, "Integrating users into the wider broadband network via high altitude platforms," IEEE Wireless Communications, vol. 12, pp. 98-105, 2005.

[23] S. H. Alsamhi, "Quality of service (QoS) Enhancement Techniques in High Altitude Platform Based Communication Networks " Ph.D, Electronics Engineering Indian Institute of Technology (Banaras Hindu University), 2015.

[24] S. R. Kassa, K. Barman, and D. Kosale, "A most promising HAPs technology for next generation wireless communication systems," in Proceedings of the 4th National Conference, 2010, pp. 1-6.

[25] P. Bilaye, V. N. Gawande, U. B. Desai, A. A. Raina, and R. S. Pant, "Low Cost Wireless Internet Access for Rural Areas using Tethered Aerostats," in Industrial and Information Systems, 2008. ICIIS 2008. IEEE Region 10 and the Third international Conference on, 2008, pp. 1-5.

[26] N. Komerath, "An imaging, communications and beamed power architecture for first responders," in Proceedings of the 1st International Conference on Wireless Technologies for Humanitarian Relief, 2011, pp. 421-428.

[27] K. Gomez, A. Hourani, L. Goratti, R. Riggio, S. Kandeepan, and I. Bucaille, "Capacity evaluation of aerial LTE base-stations for public safety communications," in Networks and Communications (EuCNC), 2015 European Conference on, 2015, pp. 133-138.

[28] Y. Wang, C. Yin, and R. Sun, "Hybrid Satellite-Aerial-Terrestrial Networks for Public Safety," in Personal Satellite Services. Next-Generation Satellite Networking and Communication Systems: 6th International Conference, PSATS 2014, Genoa, Italy, July 28-29, 2014, Revised Selected Papers, 2016, pp. 106-113.

[29] Disaster Emergency Communications is available in: http://www.fema.gov/disaster-emergency-communications.

[30] A. Tauqeer, Y. Shahid, and C. S. Rasool, "Link Fault tolerable Network Topology for Network services provision in Disaster area," Research Journal of Recent Sciences, vol. 3, pp. 58-68, 2014.

[31] L. Reynaud, T. Rasheed, and S. Kandeepan, "An integrated Aerial Telecommunications Network that supports emergency traffic," in Wireless Personal Multimedia Communications (WPMC), 2011 14th International Symposium on, 2011, pp. 1-5.

[32] Emergency Communications is available in : https://www.fcc.gov/consumers/guides/emergency-communications. 